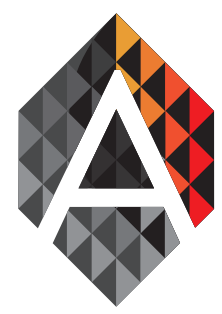

\title{
Predicting Plan Failure by Monitoring Action Sequences and Duration
}

\author{
Giovani P. Farias ${ }^{\mathrm{a}}$, Ramon Fraga Pereira ${ }^{\mathrm{a}}$, Lucas W. Hilgert ${ }^{\mathrm{a}}$, \\ Felipe Meneguzzi ${ }^{\mathrm{a}}$, Renata Vieira ${ }^{\mathrm{a}}$, and Rafael H. Bordini ${ }^{\mathrm{a}}$
}

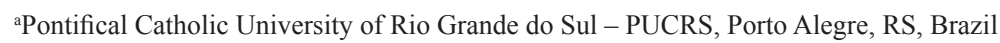
\{giovani.farias, ramon.pereira\}@acad.pucrs.br, lucaswhilgert@gmail.com, \{felipe.meneguzzi, renata.vieira, rafael.bordini\}@pucrs.br

\begin{tabular}{|c|c|}
\hline KEYWORD & ABSTRACT \\
\hline $\begin{array}{l}\text { Failure } \\
\text { Prediction; Plan } \\
\text { Recognition; } \\
\text { Multi-agent } \\
\text { System }\end{array}$ & $\begin{array}{l}\text { Anticipating failures in agent plan execution is important to enable an agent to } \\
\text { develop strategies to avoid or circumvent such failures, allowing the agent to achieve } \\
\text { its goal. Plan recognition can be used to infer which plans are being executed } \\
\text { from observations of sequences of activities being performed by an agent. In this } \\
\text { work, we use this symbolic plan recognition algorithm to find out which plan the } \\
\text { agent is performing and develop a failure prediction system, based on plan library } \\
\text { information and in simplified calendar that manages the goals the agent has to } \\
\text { achieve. This failure predictor is able to monitor the sequence of agent actions } \\
\text { and detect if an action is taking too long or does not match the plan that the agent } \\
\text { was expected to perform. We showcase this approach successfully in a health-care } \\
\text { prototype system. }\end{array}$ \\
\hline
\end{tabular}

\section{Introduction}

Recently, the number of real-world applications that deal with the need to recognise goals and plans from agent observations is on the rise. These applications can be found in fields such as human assisted living (Masato, 2012), interface agent systems (Armentano and Amandi, 2007), human-computer interaction (Hong, 2001), traffic monitoring (Pynadath and Wellman, 1995), and others. However, techniques that include the task of anticipating failures during agent plan execution have received relatively little attention. Multi-agent environments are dynamic since they are in a constant estate of change resulting from agents' actions. When these changes occur, a plan that was expected to work before, may fail. Thus, anticipating from agent observations when a plan is going to fail can be an important mechanism during the plan recognition process. Plan recognition approaches often do not make such inferences, which means that when an agent has no intention to complete or finish a plan these approaches continuously attempt to recognise what the agent is doing. In daily activities most people interrupt the plan that they are performing for some reason, such as, getting their attention drawn to something else, getting distracted by other events, or being interrupted by an emergency that needs immediate attention. In a plan recognition context, we consider that a plan is going to fail when the sequence of actions is taking too long or does not match the plan which the observed agent should be performing at the moment. Our approach uses a calendar for managing some of the agent's goals over the near future, and when that

Giovani P. Farias, Ramon Fraga Pereira, Lucas W. Hilgert, Felipe Meneguzzi, Renata Vieira, and Rafael H. Bordini Predicting Plan Failure by Monitoring Action Sequences and Duration
ADCAIJ: Advances in Distributed Computing and Articial Intelligence Journal Regular Issue, Vol. 6 N. 4 (2017), 55-69 eISSN: 2255-2863 - http://adcaij.usal.es Ediciones Universidad de Salamanca - CC BY NC DC 
information is available it facilitates our failure checking procedure, as well as plan recognition disambiguation. A plan failure can occur when an agent interrupts its current plan execution due to concurrent plans that need attention, or when an agent has to deal with conflicting plans. In this case, a mechanism to anticipate failures during agent plan execution can be useful in several situations, for example, helping an agent stay focused on a particular plan, or detecting when an agent is deviating from its regular activities.

Research on planning has focused on the modelling of actions with duration and stochastic outcomes, both theoretically as variants of Markov Decision Processes (MDP) (Mausam and Weld, 2008), and domain description languages that express temporal planning (e.g., PDDL 2.1 (Fox and Long, 2003), an extension of PDDL. In the literature, a similar approach to failure prediction, as we introduce in this work, is plan abandonment detection. Geib and Goldman (Geib and Goldman, 2003) proposes a formal model to recognise goal/plan abandonment in the plan recognition context, based on the PHATT (Probabilistic Hostile Agent Task Tracker) model (Goldman et al., 1999). This formal model estimates the probability that a set of observed actions in sequence contributes to the goal being monitored, furthermore, Geib (Geib, 2002) addresses some issues and requirements for dealing with plan abandonment, as well as intention recognition in the elderly-care domain.

In this work, we develop an approach to predict plan failure by monitoring agent's actions during its plan execution. Essentially, our approach to plan failure prediction features a mechanism that is composed of three modules. The first module is responsible for recognising the plan that the observed agent is executing. The second module checks if plans assigned to observed agent are being executed as scheduled in a predefined calendar. Lastly, the third module checks if actions are being executed as expected (i.e., not taking too long, and matching the current monitored plan). Thus, this approach can be used in complex software system, including health-care applications to improve functionality, such as activity recognition (Pereira et al., 2017; Granada et al., 2017b) and task reallocation (Panisson et al., 2015) among agents representing human users, who collaborate to take care of a patient, by detecting if a person responsible for some activity of the patient is following his scheduled appointments; detecting problems, that may prevent the person in charge to attend to his obligations and send warning to the system.

\section{Plan Recognition}

Plan recognition can be defined as the task of recognising the intentions of an agent based on the available evidence, that is, agent actions, explicit statements about intentions, and agent preferences (Kautz and Allen, 1986). Plan recognition focuses on mechanisms for recognising the unobservable state of an agent, given observations of its interaction with its environment. In other words, a plan recognition system must have a mechanism that is capable of inferring agent intentions by observing the agent actions in the environment. This mechanism retrieves, from a given set of observations, one or more hypotheses of the agent's current plan of action. The practical knowledge used to infer plans is domain dependent and, therefore, is commonly specified beforehand for each specific domain. This domain dependent information is usually encoded as two parts of inputs for the recogniser: a set of observed actions and a set of plans and goals. More specifically, the inputs to a plan recogniser are a set of goals that the recogniser expects the agent to carry out in the domain, a set of plans describing the way in which the agent can reach each goal, and a sequence of actions currently being performed by the observed agent (i.e, observations of agent actions). So, the plan recognition process itself consists in inferring the agent plan and determining how the observed actions contribute to performing it.

Plan recognition systems can be classified according to the role that an observed agent plays in the plan recognition process (Waern, 1996) into intended, keyhole, and obstructed plan recognition. In intended recognition, the observed agent is aware of the recognition process and actively participates giving signals to be sensed by the observer. An example of intended plan recognition can be found in a cooperative problem-solving environment. In keyhole recognition the user is unaware of the recognition process, which may provide only partial observability to the observer bringing the need to identify the context on its own. In a UNIX console, for example, users enter a sequence of commands to achieve a higher-level goal; an agent should infer the user's intention by observing the entered commands. In this case, the agent is following a keyhole plan recognition process. Finally, the other position that an agent might assume in a plan recognition process is obstructive. In this

Giovani P. Farias, Ramon Fraga Pereira, Lucas W. Hilgert, Felipe Meneguzzi, Renata Vieira, and Rafael H. Bordini Predicting Plan Failure by Monitoring Action Sequences and Duration
ADCAIJ: Advances in Distributed Computing and Articial Intelligence Journal Regular Issue, Vol. 6 N. 4 (2017), 55-69 eISSN: 2255-2863 - http://adcaij.usal.es Ediciones Universidad de Salamanca - CC BY NC DC 
case the user is aware of and obstructs the plan recognition process. This is a common user role in adversarial games, military and anti-terrorism scenarios. Obstructed plan recognition is applicable, for example, in a chess game, where participants do not want their opponent to recognise their intentions, and do their best to hide them.

Symbolic plan recognition is a type of plan recognition mechanism that narrows the set of candidate intentions by eliminating the plans that are incompatible with current agent actions. Plans make up a plan library and can include preconditions, effects, and sub-goals. Generally, symbolic approaches assume that the observer has complete knowledge of the agent's possible plans and goals. Symbolic approaches handle the problem of plan recognition by determining which set of goals is consistent with the observed actions. Algorithms to recognise the intentions and plans executed by autonomous agents have long been studied in the Artificial Intelligence field under the general term of plan recognition. Such work has yielded a number of approaches to plan recognition (Baker et al., 2009; Ramírez and Geffner, 2009) and models that use them in specific applications (Sukthankar and Sycara, 2011; Oh et al., 2013; Geib and Goldman, 2001). Kautz and Allen (Kautz and Allen, 1986) focus on symbolic methods providing a formal theory of plan recognition. Usually, these approaches specify a plan library as an action hierarchy in which plans are represented as a plan graph with top-level actions as root nodes, and plan recognition is then reduced to a graph covering problem. The plan recognition process attempts to find a minimal set of top plans that explain the observations.

In a wide variety of applications, plan-recognition algorithms play a crucial role, such as human-robot interaction (Cirillo et al., 2010), video surveillance (Hongeng and Nevatia, 2003), smart homes (Augusto and Nugent, 2006), intelligent user interfaces (Ullmer and Ishii, 2000), and personal agent assistants (Geib, 2002). Plan recognition has been extensively used in many other areas related or not with computer sciences including collaborative planning (Huber and Durfee, 1993), adversarial planning (Azarewicz et al., 1989), discourse analysis (Grosz and Sidner, 1990), story understanding (Charniak and Goldman, 1993; Albrecht et al., 1998), intelligent tutoring (Greer and Koehn, 1995), interface and collaborative agents (Lesh et al., 1999; Rich and Sidner, 1997; Brown, 1998), help systems (Breuker, 1990; Horvitz et al., 1998), and games (Albrecht et al., 1998). For a good overview of plan recognition in general, see Carberry (Carberry, 2001), and for the most recent research in the field of plan, intention, and activity recognition, see Sukthankar et al. (Sukthankar et al., 2014).

\subsection{Knowledge Base}

Most plan recognition systems require a knowledge base that encodes into recipes the ways in which agent goals can be achieved. A plan library is a knowledge base that codifies in some way the agent's beliefs concerning how the agent can reach each particular goal in the domain. Plan recognition systems have a plan library as an input, so several representations of agent plans have been used to approach this problem, and various methods applied to infer the agent's intention. These methods can be grouped in two main categories: symbolic and probabilistic approaches. Symbolic approaches aim at narrowing the set of candidate intentions by eliminating those plans that cannot be explained by the actions that the agent performs. The most used representation for symbolic approaches are plan hierarchies and consistency graphs. Probabilistic approaches explicitly represent the uncertainty associated with agent plans and allow a probabilistic ranking of the agent intentions mainly making use of Bayesian Networks (Pearl, 1988) and Markov Models (Bui and et al., 2004; Bui et al., 2002). Most symbolic and probabilistic approaches are domain independent and can lead to accurate predictions provided the plan library is complete (for symbolic approaches) or provided the probabilities are correct (for probabilistic approaches). These approaches normally have the disadvantage of considering all the possible plans in the plan library given the observations. However, if observations so far cannot distinguish between a set of possible intentions, probabilistic approaches can find the most probable one, while symbolic approaches can not select between them and have to wait for a single consistent explanation. Symbolic approaches are very sensitive to noisy actions, as the plan recogniser could wrongly exclude a plan (from the hypotheses explaining the observed behaviour) if an unexpected action occurs in the middle of the execution of a plan.

Many plan recognition systems structure their plan libraries as an Hierarchical Task Network (HTN) (Erol et al., 1994b; Erol et al., 1994a) to define the set of plans they are expected to recognise, in which goals are the root nodes and the observed actions are directly mapped to the leaf nodes. An attachment point in an HTN tree is a point in which an observation can be assigned to an action not observed yet, while shared leaders are action

Giovani P. Farias, Ramon Fraga Pereira, Lucas W. Hilgert, Felipe Meneguzzi, Renata Vieira, and Rafael H. Bordini Predicting Plan Failure by Monitoring Action Sequences and Duration
ADCAIJ: Advances in Distributed Computing and Articial Intelligence Journal Regular Issue, Vol. 6 N. 4 (2017), 55-69 eISSN: 2255-2863 - http://adcaij.usal.es Ediciones Universidad de Salamanca - CC BY NC DC 
prefixes in the plan library that are common to different plans with different goals (root nodes). Kautz and Allen (Kautz and Allen, 1986) describe the system knowledge about agent actions in terms of a hierarchy of specialisation and decomposition events, where decomposition captures the sub goals that are specific to an action and specialisation represents the different ways of performing a more general action. They specified the plan library as an action hierarchy in which plans are represented in a plan graph with top-level actions as root nodes, and plan recognition process is then a problem of graph covering. McCarthy's circumscription (McCarthy, 1986) was used to transform the hierarchy by circumscribing the ways of specialising an act, and then circumscribing the ways of using an act, closing the action hierarchy. From the action hierarchy, a set of axioms from which all desired conclusions could be derived deductively is specified. Brown (Brown, 1998) uses Bayesian networks to build an agent profile that allows the detection of its intentions considering the utility of offering assistance. This approach represents the causality between preconditions, goals, and actions in terms of an AND/OR graph composing a goal hierarchy to represent user intentions. Goldman, Geib, and Miller (Goldman et al., 1999) assume that a plan library is made up of tasks structured in an hierarchical way, in which task nodes could represent goals, methods, and primitive actions. Similarly to Brown (Brown, 1998), the plan library could be viewed as a partially ordered AND/OR tree, in which the AND nodes are methods, connecting all action steps or sub-tasks needed to achieve the parent task, and the OR nodes are other isolated sub-tasks.

\section{Symbolic Plan Recognition}

The Symbolic Plan Recognition (SBR) (Avrahami-Zilberbrand and Kaminka, 2005) is a method for complete, symbolic plan recognition that uses a plan library, which encodes agent knowledge in the form of plans. SBR extracts coherent hypotheses from a multi-featured observation sequence using a Feature Decision Tree (FDT) to efficiently match these observations to plan steps ${ }^{1}$ in a plan library. An FDT is a decision tree, where nodes represent observable features and the branches represent conditions on their values. So, finding all matching plans, from a set of observable features, is just a matter of traversing the FDT top-down until a leaf node is reached.

\subsection{Plan Library}

A plan library is represented by a single-root directed acyclic connected graph, which includes all possible plans that an observed agent may execute. The term plan is used here in a broader sense, representing behaviours, reaction plans, and recipes. Typically, a plan library has a single root node in which its children are top-level plans and all other nodes are simply plan steps. Furthermore, in a plan library, sequential edges specify the expected temporal order of a plan execution sequence and decomposition edges decompose plan steps into alternative sub-steps. The plan library has no hierarchical cycles. However, plans may have a (sequential) self-cycle, allowing a plan step to be executed during multiple subsequent time stamps. Each agent action generates a set of conditions on observable features that are associated with that action. When these conditions are included, the observations match particular plan steps.

Figure 1 shows a plan library example based on Activities of Daily Living (ADL), which is a term used in health-care to refer to daily self-care activities of people. This plan library shows sequential links represented by dashed arrows and decomposition links represented by solid arrows. For instance, there is a decomposition link between managing-medication and getting-up, and a sequential link between getting-up and using-bathroom. The top-level plans are managing-medication, and leisure. Figure 1 does not show the set of conditions on observable features associated with plan steps, and circled numbers denote time stamps (e.g., using-bathroom has been considered a hypothesis at time stamp 2). Moreover, the path root $\rightarrow$ managing-medication $\rightarrow$ having-lunch $\rightarrow$ at-kitchen $\rightarrow$ taking-medication can be a hypothesis for the current plan being executed by an observed agent.

1. In this work, we use «plan step» as a synonym for «action».

Giovani P. Farias, Ramon Fraga Pereira, Lucas W. Hilgert, Felipe Meneguzzi, Renata Vieira, and Rafael H. Bordini Predicting Plan Failure by Monitoring Action Sequences and Duration
ADCAIJ: Advances in Distributed Computing and Articial Intelligence Journal Regular Issue, Vol. 6 N. 4 (2017), 55-69 eISSN: 2255-2863 - http://adcaij.usal.es Ediciones Universidad de Salamanca - CC BY NC DC 


\subsection{Feature Decision Tree - FDT}

The first stage of the SBR approach is the matching phase, in which the observations made by the recognizer are matched against plans in the plan library. The SBR algorithm considers complex observations presuming that each plan step has a set of conditions on observable features associated with it (Fagundes et al., 2014). When these conditions hold in regards to observed features of action execution (and in the correct order in case of sequential edges), the current observation is said to match that plan. Matching observations to plans can be computationally expensive if all plans are checked and for each plan all observed features are also checked (Kaminka and Tambe, 2000). To speed-up this process of matching observations to plans, SBR augments

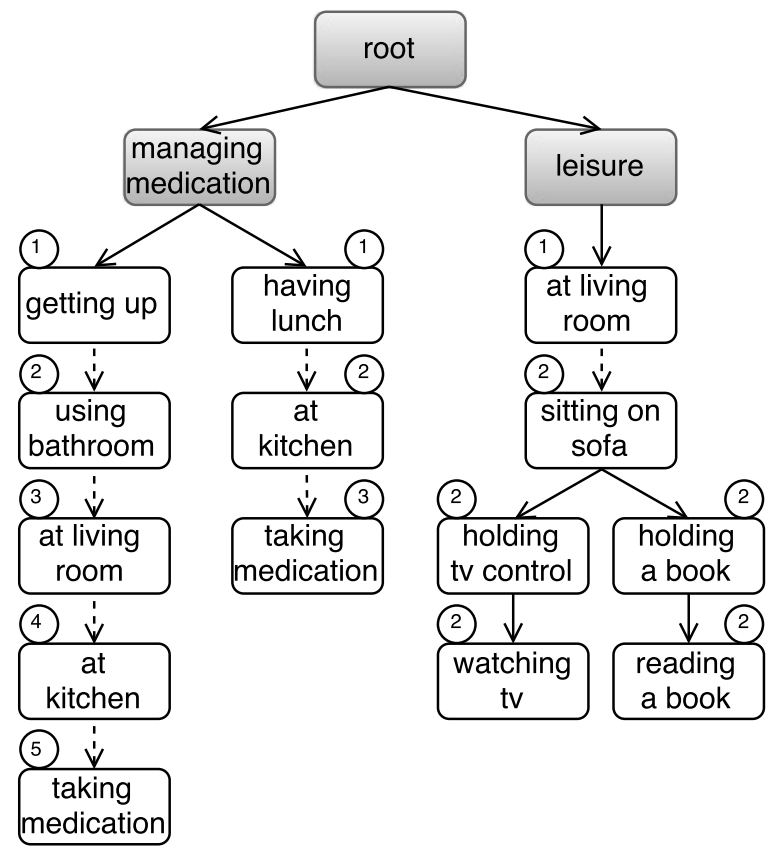

Figure 1: Example of plan library based on $A D L$

the plan library with a Feature Decision Tree (FDT) data structure, which efficiently maps observations to matching nodes in the plan library. An FDT is a decision tree, where each node represents an observable feature and each branch represents one possible value of this feature. Determining all matching plans, from a set of observations features, is efficiently achieved by traversing the FDT top-down until a leaf node is reached. Each leaf node is a pointer to a plan step in the plan library. The connection between FDT and plan library is shown in Figure 2, which shows part of an FDT using features associated with plan steps in Figure 1. A plan step executed by an agent can be identified according to observed features, and these features values are used by the FDT to separate the plan steps. To determine matching plan steps, the matching algorithm first checks FDT root nodes, which in Figure 2 are represented by the «has_location_change» feature. The algorithm then (based on feature value) follows the appropriate branch to test in sequence other features until a leaf node is reached. Thus, each leaf node will have pointers to all instance of the plan steps associated with it in the plan library (Avrahami-Zilberbrand, 2009).

Giovani P. Farias, Ramon Fraga Pereira, Lucas W. Hilgert, Felipe Meneguzzi, Renata Vieira, and Rafael H. Bordini Predicting Plan Failure by Monitoring Action Sequences and Duration
ADCAIJ: Advances in Distributed Computing and Articial Intelligence Journal Regular Issue, Vol. 6 N. 4 (2017), 55-69 eISSN: 2255-2863 - http://adcaij.usal.es Ediciones Universidad de Salamanca - CC BY NC DC 


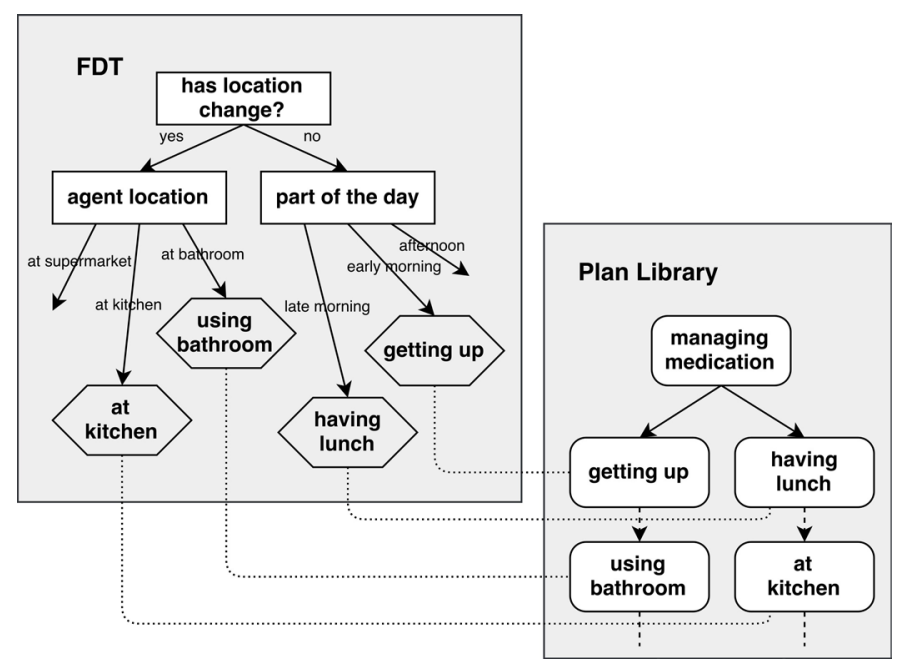

Figure 2: Example of an FDT associated with a plan library

\section{Failure Predictor Components}

The failure predictor is responsible for predicting plan failures during execution of an agent goal, more specifically, it tracks the execution of a goal and attempts to identify elements which can lead it to fail, for example, an action taking significantly more time than expected to conclude. The failure predictor is composed of three modules: the SBR module, which is responsible for recognising the plan that is being executed by the observed agent; an Appointment Controller module, which checks if the goals that are known to have been assigned to the agent are being executed as scheduled; and a Plan-Step Controller module, that checks if the plan steps (that compose the plan) are being executed as expected. These modules are detailed, respectively, in Subsections 4.1, 4.2 , and 4.3 .

\subsection{SBR Component}

The SBR component implements the symbolic recogniser presented in Section 3. It is responsible for recognising the plan that an agent is currently executing, and it generates hypotheses about possible plans while the recognition is still not possible. This information is represented both as a list of candidate plans and as a hypotheses graph. As input, the SBR component receives observations, i.e., sets of contextual information about the observed agent and its actions. Examples of observations include the agent's global positioning coordinates, whether or not the agent is moving, or whether the agent is approaching a particular place, and any other contextual information that can be generated by an activity recognition process (Granada et al., 2017a). As output, this component provides both the list of candidate plans and the hypotheses graph.

\subsection{Appointment Controller}

A plan library contains all known plans (agent goals) for a given domain, together with the sequence of actions that compose them, however, it does not define the time that an agent is expected to execute each plan, neither does it contain the time interval in which the plans have to be executed. These are essential information for the system to ensure that plans are being executed in an appropriate manner and to be able to detect potential failures in plan execution. The Appointment Controller component implements a simplified calendar which manages the agent goals and plans, it defines which plans of the plan library an agent is known to be responsible for, and at which time the agent is expected to execute some of them (to the extent that this is known in particular domains). This component also helps in disambiguation of candidate plans and in the early

Giovani P. Farias, Ramon Fraga Pereira, Lucas W. Hilgert, Felipe Meneguzzi, Renata Vieira, and Rafael H. Bordini Predicting Plan Failure by Monitoring Action Sequences and Duration
ADCAIJ: Advances in Distributed Computing and Articial Intelligence Journal Regular Issue, Vol. 6 N. 4 (2017), 55-69 eISSN: 2255-2863 - http://adcaij.usal.es Ediciones Universidad de Salamanca - CC BY NC DC 
prediction of plan failures. It should be noted that only domain-related plans are kept in this individual calendar. Each entry (i.e., agent goal) in the Appointment Controller is composed of the following information:

- starting date - date in which the goal or plan is expected to be started;

- ending date - date in which the goal or plan is expected to end;

- title - title of goal or plan (e.g., «managing-medication»);

- description (optional) - brief textual description of the goal (e.g., «take medicine on time»);

- related plan id - unique identifier of the relevant plan (i.e., the plan to achieve this goal), which corresponds to a top plan in the plan library (e.g., «id:p1»);

- tolerance - margin of error for the beginning and end times of each goal, e.g., some goals can start or end 5 minutes before (or after) the time for which it was originally scheduled without danger of the plan failing. This tolerance is necessary because, in real-world situations, goals usually do not start (or end) at the exact scheduled time.

Regarding schedule times, both the starting and ending dates of the goals are composed of day, month, year, hour, and minute (smaller units such as second, for example, are not necessary). The tolerance interval can be expressed in various time measures (e.g., hours or minutes).

\subsection{Plan-Step Controller}

The Plan-Step Controller monitors and analyses the plan execution (sequence of actions) to detect anomalies that can lead the plan to fail. The information necessary for the Plan-Step Controller to operate is obtained through the SBR component, which provides information about the current plan and actions being performed; the plan library; and the file that contains information about expected time for each action execution. The plan library contains the known plans and the actions which need to be performed in a given plan for it finish successfully, besides the sequence in which these actions must be performed for a plan to be considered completed. However, it does not define when a plan should finish, neither the time in which actions must be executed. This type of information is important to detecting anomalous behaviour during plan execution, such as:

- plan interruption - the plan execution is interrupted without all actions being completed;

- time exceeded - when an action takes significantly more time than expected, this typically leads to plan failure (e.g., being in a traffic jam);

- inconsistent sequence - the sequence of observed actions is inconsistent with the expected plan path in the plan library.

It is important to keep track of the actions being performed in order to be able to predict whether a plan is following the expected execution path. Thus, it is possible to identify a probable failure in plan execution and generate the required warnings according to failure type. The entry in file with data about expected execution time of each action is composed of the following information:

- plan-step id - unique identifier, which corresponds to a plan step in the plan library (e.g., «p1.11»);

- label - a label for identification the action (plan step) from the plan library (e.g., «taking-medication»);

- time - time that an action often take to be performed;

- tolerance - a value whereby the ending time of the action is allowed to be delayed. For instance, an action can take 5 minutes in addition to its normal time to be performed. This tolerance is important for a real-world situation where actions can often take more time to be performed than an exact specified time.

Giovani P. Farias, Ramon Fraga Pereira, Lucas W. Hilgert, Felipe Meneguzzi, Renata Vieira, and Rafael H. Bordini Predicting Plan Failure by Monitoring Action Sequences and Duration
ADCAIJ: Advances in Distributed Computing and Articial Intelligence Journal Regular Issue, Vol. 6 N. 4 (2017), 55-69 eISSN: 2255-2863 - http://adcaij.usal.es Ediciones Universidad de Salamanca - CC BY NC DC 


\section{Integration of the Components}

We illustrate the integration of the failure predictor components in Figure 3. When the SBR (Section 3) is not able to determine the current plan (no plan or multiple plans were recognised) the Appointment Controller component is consulted (using the output of the plan recogniser). First, the component checks if there are plans scheduled for the moment in which it was consulted and, later, if a scheduled plan is in the list of candidate plans. During this verification the following situations might occur:

- There is no agent goal scheduled for the current time. In this situation, the Appointment Controller component has nothing to do, so the main cycle ends and the system awaits for a new observation;

- There is a plan scheduled for the current time, however, the candidate plan list is empty (no plans were recognised). In this case, the Appointment Controller component detects a failure in the scheduled goal execution (i.e., the goal that was expected to be executed at time the calendar was consulted) and a warning must be sent to the system, which should be able to handle this plan failure;

- There is a plan scheduled for the current time and there are multiple plans in candidate plans list. In this case, the Appointment Controller verifies if the plan related to scheduled goal is present in plan candidate list. During this verification two situations might happen:

- The plan related to the scheduled goal is in candidate plans list, thus, the referred plan is assumed to be the one that is currently being executed by agent;

- The plan related to the scheduled goal is not in candidate plans list. In this situation, a failure is detected in the scheduled goal execution as it is not being executed by the agent as it should. Then, a warning related to the scheduled goal is sent to the Handling Plan Inconsistency step of the main cycle, in which the system will handle the plan related to the failing goal.

Regarding, goal scheduling in this initial implementation does not allow overlapping of goals (i.e., goals with coincident time intervals). That is, a new goal will not be added to calendar if it overlaps with an existing one.

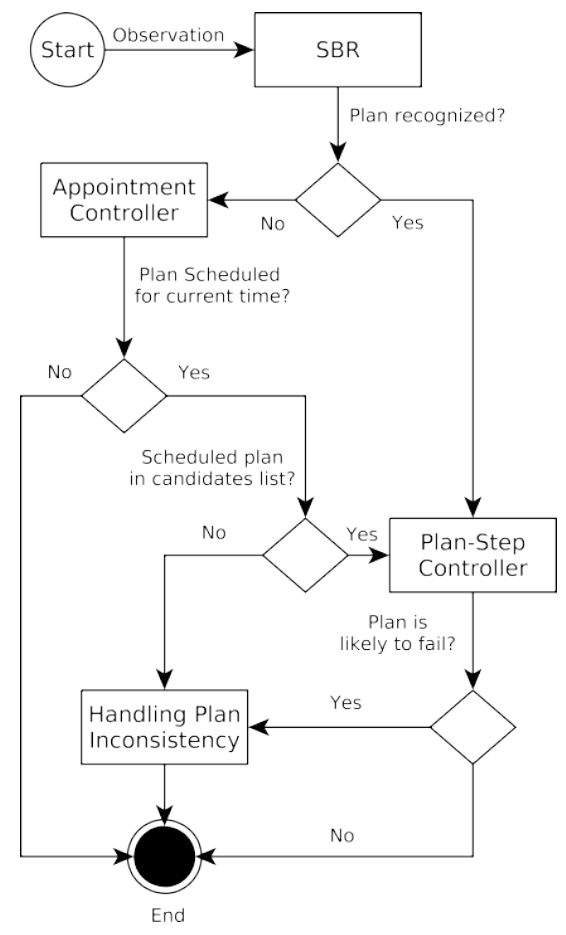

Figure 3: Components Integration

Giovani P. Farias, Ramon Fraga Pereira, Lucas W. Hilgert, Felipe Meneguzzi, Renata Vieira, and Rafael H. Bordini Predicting Plan Failure by Monitoring Action Sequences and Duration
ADCAIJ: Advances in Distributed Computing and Articial Intelligence Journal Regular Issue, Vol. 6 N. 4 (2017), 55-69 eISSN: 2255-2863 - http://adcaij.usal.es Ediciones Universidad de Salamanca - CC BY NC DC 
However, there is an exception for the overlapping rule regarding the starting and ending times. Two goals ( $A$ and $B$ ) are not considered as overlapping if the starting time of $A$ is equal to the ending time of $B$. This exception is convenient, as sequential plans are usually scheduled with no time interval among them, e.g., $A$ (1:00 p.m. to 2:00 p.m.) and $B(2: 00$ p.m. to 4:00 p.m.) or $A$ (4:00 p.m. to 5:00 p.m.) and $B(2: 00$ p.m. to 4:00 p.m.).

The Plan-Step Controller is unable to disambiguate the list of candidate plans, thus, both SBR and Appointment Controller must inform only one goal and one plan step in each iteration with it. The planController (Algorithm 1) is part of this component and responsible for handling such information, using the plan library and the data file with the plan step running times, in order to analyse the plan sequence (based on plan steps) and monitor the running time of each action. In this manner, it is possible to detect and report possible changes in plan execution in order to avoid possible failures.

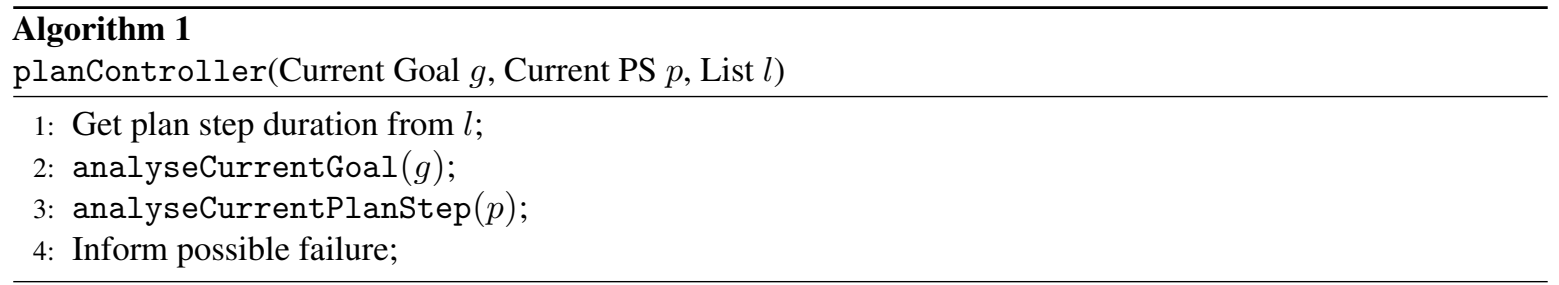

Monitoring of the current plan is performed by the analyseCurrentGoal (Algorithm 2). Initially, the current goal is updated based on information received by SBR (Line 1) and a test is performed to check if it is a valid value (Line 2), after that, the algorithm checks if the current goal is equal to the previous goal (Line 5) that the agent was trying to achieve. If they are the same, it means agent is still carrying out the actions to achieve it, otherwise, the agent started to perform a new goal with a new plan. In this case, the algorithm has to verify if the previous goal was achieved successfully (Line 11) and the information in the plan library is used to check if the last plan step (of the previous goal) is a leaf node. If this is the case, it means that the plan was fully executed and probably has finished successfully, otherwise, the agent may have stopped performing the plan before its end or the agent is executing more than one plan at the same time, thus, the algorithm should send a warning about this possible failure (Line 15).

The analyseCurrentPlanStep (Algorithm 3) is responsible for monitoring the execution of each action related to a goal, i.e., it analyses the sequence of execution and the run time of each plan step. Initially, the current plan step is updated based on information received by SBR. The consistency of this information is checked and the algorithm then checks if the current plan step is equal to the previous plan step which the agent was performing (Line 5). If they are equal, it means that the agent is still performing the same plan step, thus, the algorithm has to check if the current action is within the time specified in the data file with the plan step running times. The checkExecutionTime (Line 7) receives as input the current plan step and checks if its run time is within the specified time, taking into account the specified tolerance for each action. If the agent is taking too long to perform some action, the algorithm detects this as unexpected behaviour and warns the system.

When the failure predictor detects a new action being performed by an agent, it is necessary to check if this action is part of the sequence of actions needed to accomplish the current goal (Line 9). The isValidsequence (Algorithm 4) uses the information in the plan library to check if the current plan step is part of a valid sequence

Giovani P. Farias, Ramon Fraga Pereira, Lucas W. Hilgert, Felipe Meneguzzi, Renata Vieira, and Rafael H. Bordini Predicting Plan Failure by Monitoring Action Sequences and Duration
ADCAIJ: Advances in Distributed Computing and Articial Intelligence Journal Regular Issue, Vol. 6 N. 4 (2017), 55-69 eISSN: 2255-2863 - http://adcaij.usal.es Ediciones Universidad de Salamanca - CC BY NC DC 

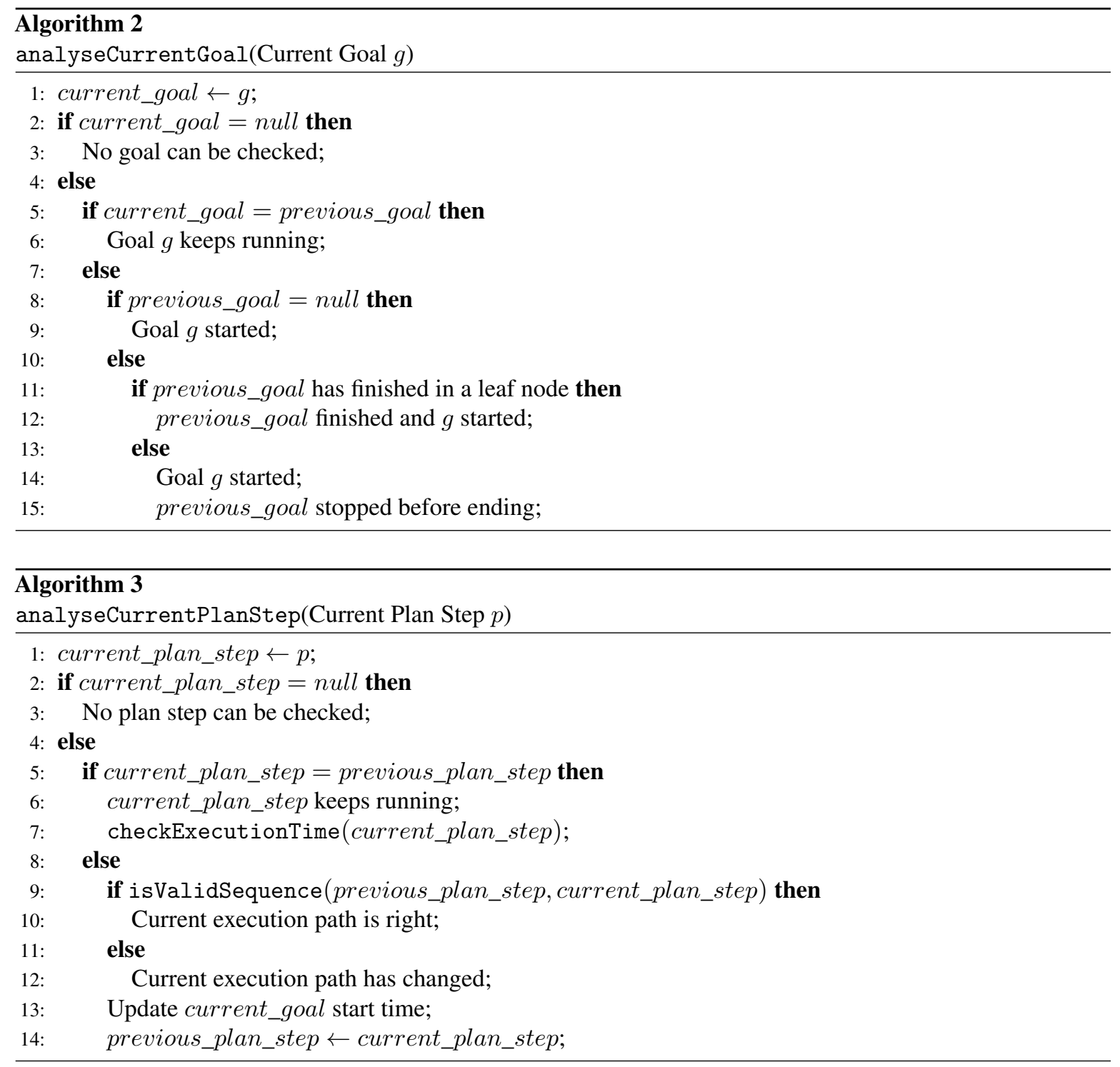

of actions to achieve the current goal, receiving as input the previous plan step and the current plan step. If the current plan step contains a sequential parent node and this parent node is the previous plan step, it means the execution path is correct, otherwise, the current plan step does not match the execution path done so far to achieve the current goal.

Giovani P. Farias, Ramon Fraga Pereira, Lucas W. Hilgert, Felipe Meneguzzi, Renata Vieira, and Rafael H. Bordini Predicting Plan Failure by Monitoring Action Sequences and Duration
ADCAIJ: Advances in Distributed Computing and Articial Intelligence Journal Regular Issue, Vol. 6 N. 4 (2017), 55-69 elSSN: 2255-2863 - http://adcaij.usal.es Ediciones Universidad de Salamanca - CC BY NC DC 


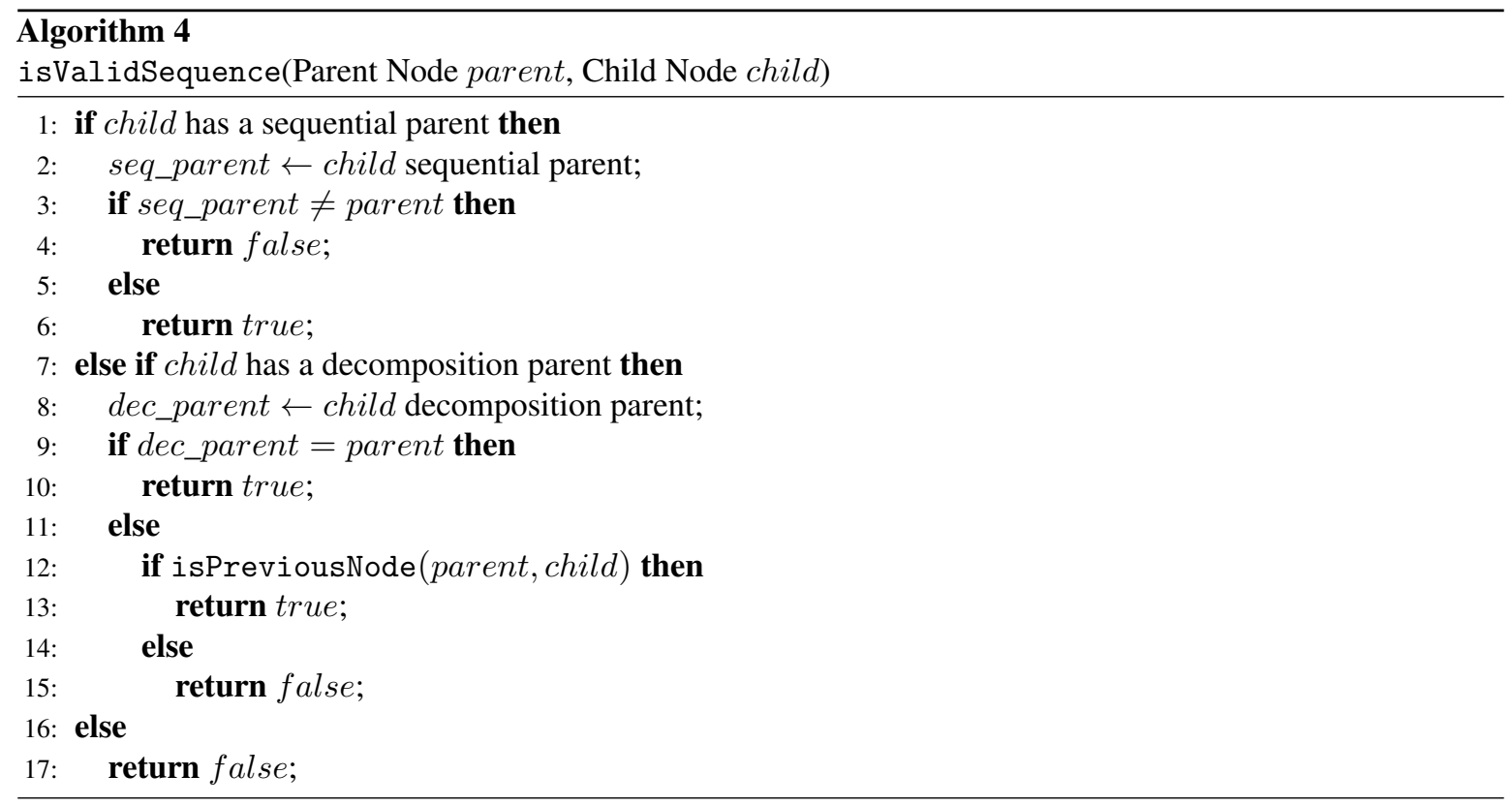

Detecting whether the sequence of execution is valid is more complicated when the current plan step has a decomposition parent node, because the previous plan step does not have to be a parent node of the current plan step, but only be part of the current plan execution and follow the temporal order of the execution path. The isPreviousNode (Algorithm 5) algorithm receives as input the previous plan step and the current plan step. It checks the entire running sequence from current plan step node to previous plan step node in order to analyse the temporal order to determine if the current plan step is a valid sequence for current goal execution.

\section{Experiments}

The objective of experiments is to show how our approach provides helpful reminders by monitoring and anticipating plan failure from agent observations, in this way, we model part of a scenario that represents agent behaviour based on Activities of Daily Living. These activities correspond to user single actions (e.g., getting-up, watching-tv, reading-a-book, taking-medication, using-bathroom), in this scenario, there is a person with disabilities who lives alone and needs constant monitoring to perform his daily activities, using plan library

Giovani P. Farias, Ramon Fraga Pereira, Lucas W. Hilgert, Felipe Meneguzzi, Renata Vieira, and Rafael H. Bordini Predicting Plan Failure by Monitoring Action Sequences and Duration
ADCAIJ: Advances in Distributed Computing and Articial Intelligence Journal Regular Issue, Vol. 6 N. 4 (2017), 55-69 eISSN: 2255-2863 - http://adcaij.usal.es Ediciones Universidad de Salamanca - CC BY NC DC 


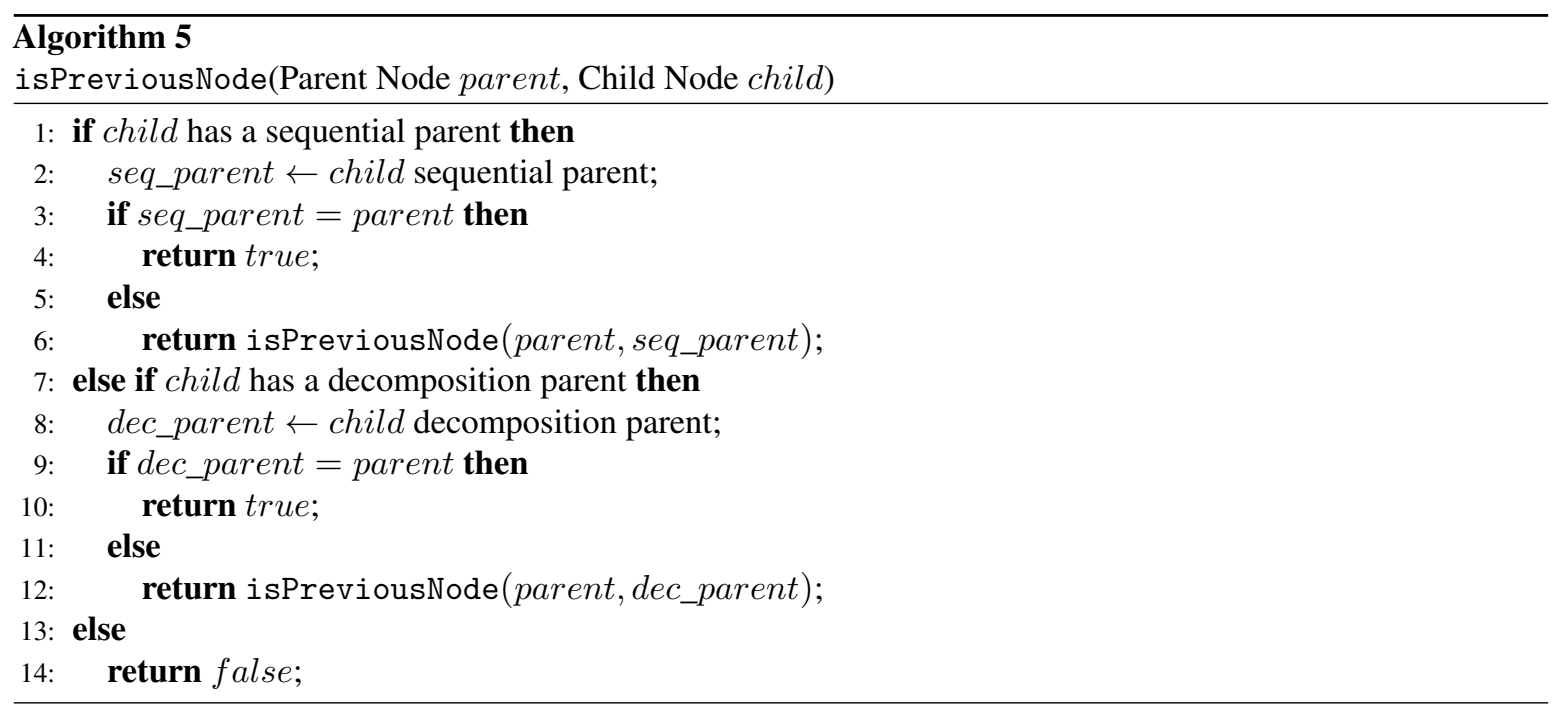

formalism, we model a set of plans for representing possible behaviour of this person, where some of these plans are shown in Figure 1.

To exemplify how our approach works, we schedule the top-level plan managing-medication to be performed between 7:00 a.m. and 7:30 a.m., in which, this schedule information is in the calendar (Appointment Controller). Considering the current part of the day as early morning, the sequence of activities to be performed in order to accomplish the plan managing-medication is: getting-up $\rightarrow$ using-bathroom $\rightarrow$ at-living-room $\rightarrow$ at-kitchen $\rightarrow$ taking-medication. According to this sequence, the user must move through the living room (i.e., plan step at-living-room) to complete the plan, however, this plan step is also part of the top-level plan leisure, in this case, the SBR component returns both top-level plans managing-medication and leisure when the current plan step is at-living-room. To deal with this ambiguity, our approach uses the Appointment Controller component to check if there is a top-level plan scheduled for the current time, if so, it discards those plans that are not scheduled for this time.

The program output, presented in Table 1, represents part of execution of the failure predictor approach, in a scenario where the user must take a medication in a strict time and during the plan execution the user's attention drawn to something else (e.g., the user stays at living room watching TV) and forgets to take his medication. In this case, the current top-level plan is managing-medication (Line 2) and the current plan step is at-livingroom (Line 3). The failure predictor monitors the plan execution (Lines 4-6) and based on information in the Plan-Step Controller, i.e., average time of execution for each plan step and a time tolerance (Line 7), it is able to detect anomalies in plan execution and informs the system to try to address and correct these possible failures. In this example, the algorithm detects that a plan step is taking too long to be performed, then an alert message is generated (Line 8).

Table 1: Example of Failure Predictor Output

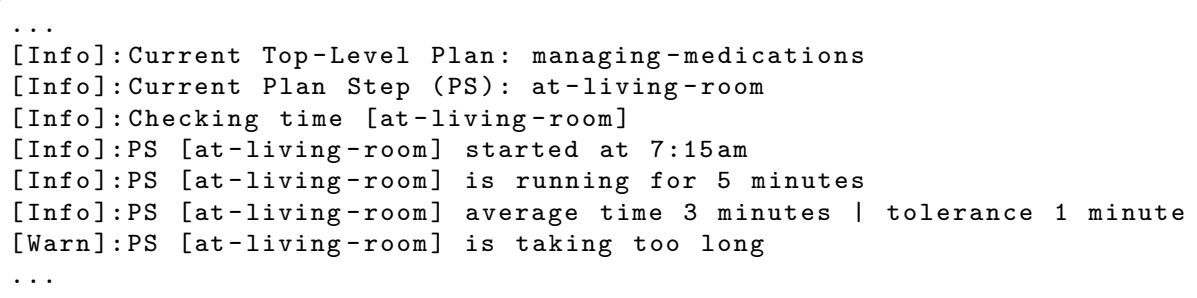

Giovani P. Farias, Ramon Fraga Pereira, Lucas W. Hilgert, Felipe Meneguzzi, Renata Vieira, and Rafael H. Bordini Predicting Plan Failure by Monitoring Action Sequences and Duration
ADCAIJ: Advances in Distributed Computing and Articial Intelligence Journal Regular Issue, Vol. 6 N. 4 (2017), 55-69 eISSN: 2255-2863 - http://adcaij.usal.es Ediciones Universidad de Salamanca - CC BY NC DC 


\section{Conclusion}

In this work, we have developed a failure predictor based on plan recognition techniques and a calendar that includes some of the plans that the agent is known to be required to execute over time. Our main contribution is a system that anticipates plan failures by monitoring a sequence of agent actions during its plan execution. We have used this predictor as part of a system to support collaborative work in a scenario where family members and professional carers support an elderly person with a debilitating disease who lives alone. Although we currently only deal with plan failure prediction, as future work we can enable our system to elaborate alternative plans to avoid the detected failures (e.g., using planning techniques) rather than simply warning about possible plan failures.

\section{Acknowledgements}

Part of the results of this work were obtained through the research project entitled «Semantic and Multi-Agent Technologies for Group Interaction», sponsored by Samsung Eletrônica da Amazônia Ltda. under the terms of Brazilian federal law No. 8.248/91.

\section{References}

Albrecht, D. W., Zukerman, I., and Nicholson, A. E., 1998. Bayesian Models for Keyhole Plan Recognition in an Adventure Game. User Model. User-Adapt. Interact., 8(1-2): 5-47.

Armentano, M. G. and Amandi, A., 2007. Plan Recognition for Interface Agents. Artificial Intelligence, 28(2):131-162. ISSN 0269-2821. doi:10.1007/s10462-009-9095-8.

Augusto, J. C. and Nugent, C. D., editors, 2006. Designing Smart Homes, The Role of Artificial Intelligence, volume 4008 of Lecture Notes in Computer Science. Springer. ISBN 3-540-35994-X.

Avrahami-Zilberbrand, D., 2009. Efficient Hybrid Algorithms for Plan Recognition and Detection of Suspicious and Anomalous Behavior. Ph.D. thesis, Bar Ilan University.

Avrahami-Zilberbrand, D. and Kaminka, G. A., 2005. Fast and Complete Symbolic Plan Recognition. In Kaelbling, L. P. and Saffiotti, A., editors, Proceedings of the International Joint Conference on Artificial Intelligence, pages 653-658. Professional Book Center. ISBN 0938075934.

Azarewicz, J., Fala, G., and Heithecker, C., 1989. Template-based multi-agent plan recognition for tactical situation assessment. In Fifth Conference on Artificial Intelligence Applications, pages 247-254.

Baker, C., Saxe, R., and Tenenbaum, J., 2009. Action Understanding as Inverse Planning. Cognition, 31: 329349. Breuker, J., 1990. Conceptual Model of Intelligent Help Systems. In Breuker, J., editor, EUROHELP: developing Intelligent Help Systems, chapter 2, pages 41-66. EC, Copenhagen, DM.

Brown, S. M., 1998. A Decision Theoretic Approach for Interface Agent Development. Ph.D. thesis, Air Force Institute of Technology, Wright Patterson AFB, OH, USA. AAI9905125.

Bui, H. H. and et al., 2004. Hierarchical Hidden Markov Models with General State Hierarchy. In Proceedings of the 19th national conference on artificial intelligence, pages 324-329.

Bui, H. H., Venkatesh, S., and West, G., 2002. Policy Recognition in the Abstract Hidden Markov Model. $J$. Artif. Int. Res., 17(1): 451-499. ISSN 1076-9757.

Carberry, S., 2001. Techniques for Plan Recognition. User Modeling and User-Adapted Interaction, 11(1-2): 31-48. ISSN 0924-1868.

Charniak, E. and Goldman, R. P., 1993. A Bayesian Model of Plan Recognition. Artif. Intell., 64(1): 53-79. Cirillo, M., Karlsson, L., and Saffiotti, A., 2010. Human-aware Task Planning: An Application to Mobile Robots. ACM Trans. Intell. Syst. Technol., 1(2):15: 1-15:26. ISSN 2157-6904. doi:10.1145/1869397.1869404.

Erol, K., Hendler, J., and Nau, D. S., 1994a. HTN Planning: Complexity and Expressivity. In Proceedings of the Twelfth National Conference on Artificial Intelligence (Vol. 2), AAAI'94, pages 1123-1128. American Association for Artificial Intelligence, Menlo Park, CA, USA. ISBN 0-262-61102-3.

Giovani P. Farias, Ramon Fraga Pereira, Lucas W. Hilgert, Felipe Meneguzzi, Renata Vieira, and Rafael H. Bordini Predicting Plan Failure by Monitoring Action Sequences and Duration
ADCAIJ: Advances in Distributed Computing and Articial Intelligence Journal Regular Issue, Vol. 6 N. 4 (2017), 55-69 eISSN: 2255-2863 - http://adcaij.usal.es Ediciones Universidad de Salamanca - CC BY NC DC 
Erol, K., Hendler, J. A., and Nau, D. S., 1994b. UMCP: A Sound and Complete Procedure for Hierarchical Task-network Planning. In AIPS, pages 249-254.

Fagundes, M., Meneguzzi, F., Bordini, R. H., and Vieira, R., 2014. Dealing with Ambiguity in Plan Recognition under Time Constraints. In Proceedings of the Thirteenth International Conference on Autonomous Agents and Multiagent Systems, pages 389-396.

Fox, M. and Long, D., 2003. PDDL2.1: An Extension to PDDL for Expressing Temporal Planning Domains. Journal of Artificial Intelligence Research, 20(1): 61-124. ISSN 1076-9757.

Geib, C. W., 2002. Problems with intent recognition for elder care. In Proceedings of the AAAI-02 Workshop Automation as Caregiver, pages 13-17.

Geib, C. W. and Goldman, R. P., 2001. Plan Recognition in Intrusion Detection Systems. In Proceedings of the Second DARPA Information Survivability Conference and Exposition (DISCEX II), pages 329-342.

Geib, C. W. and Goldman, R. P., 2003. Recognizing Plan/Goal Abandonment. In Proceedings of the 18th International Joint Conference on Artificial Intelligence, IJCAI'03, pages 1515-1517. Morgan Kaufmann Publishers Inc., San Francisco, CA, USA.

Goldman, R. P., Geib, C. W., and Miller, C. A., 1999. A New Model of Plan Recognition. Artificial Intelligence, 64: 53-79.

Granada, R., Monteiro, J., Barros, R. C., and Meneguzzi, F., 2017a. A Deep Neural Architecture for Kitchen Activity Recognition.

Granada, R., Pereira, R. F., Monteiro, J., Barros, R., Ruiz, D., and Meneguzzi, F., 2017b. Hybrid Activity and Plan Recognition for Video Streams. In The 2017 AAAI Workshop on Plan, Activity, and Intent Recognition. Greer, J. E. and Koehn, G. M., 1995. The Peculiarities of Plan Recognition for Intelligent Tutoring Systems. In IJCAI-95 Workshop on the Next Generation of Plan Recognition Systems, pages 54-59. Montreal, Canada.

Grosz, B. J. and Sidner, C. L., 1990. Plans for discourse. In Cohen, P., Morgan, J., and Pollack, M., editors,

Intentions in communication, pages 417-444. MIT Press.

Hong, J., 2001. Goal recognition through goal graph analysis. Journal of Artificial Intelligence Research, 15: $1-30$.

Hongeng, S. and Nevatia, R., 2003. Large-scale event detection using semi-hidden Markov models. In Computer Vision, 2003. Proceedings. Ninth IEEE International Conference on, pages 1455-1462 vol. 2. doi:10.1109/ ICCV.2003.1238661.

Horvitz, E., Breese, J., Heckerman, D., Hovel, D., and Rommelse, K., 1998. The Lumiere project: Bayesian user modeling for inferring the goals and needs of software users. In In Proceedings of the Fourteenth Conference on Uncertainty in Artificial Intelligence, pages 256-265. Madison, WI.

Huber, M. and Durfee, E. H., 1993. Observational Uncertainty in Plan Recognition Among Interacting Robots. In International joint conference on AI (IJCAI). Workshop on Dynamically Interacting Robots, pages 68-75. Kaminka, G. A. and Tambe, M., 2000. Robust Agent Teams via Socially-attentive Monitoring. J. Artif. Int. Res., 12(1): 105-147. ISSN 1076-9757.

Kautz, H. A. and Allen, J. F., 1986. Generalized Plan Recognition. In Kehler, T., editor, Proceedings of the Conference of the American Association of Artificial Intelligence (AAAI-86), pages 32-37. Morgan Kaufmann.

Lesh, N., Rich, C., and Sidner, C. L., 1999. Using Plan Recognition in Human-computer Collaboration. In Proceedings of the Seventh International Conference on User Modeling, UM '99, pages 23-32. Springer- Verlag New York, Inc., Secaucus, NJ, USA. ISBN 3-211-83151-7.

Masato, D., 2012. Incremental Activity and Plan Recognition for Human Teams. Ph.D. thesis, University of Aberdeen.

Mausam and Weld, D. S., 2008. Planning with Durative Actions in Stochastic Domains. Journal of Artificial Intelligence Research, 31(1): 33-82. ISSN 1076-9757.

McCarthy, J., 1986. Applications of Circumscription to Formalizing Common-Sense Knowledge. Artif. Intell., 28(1): 89-116.

Oh, J., Meneguzzi, F., Sycara, K. P., and Norman, T. J., 2013. Prognostic Normative Reasoning. Eng. Appl. of AI, 26(2): 863-872.

Giovani P. Farias, Ramon Fraga Pereira, Lucas W. Hilgert, Felipe Meneguzzi, Renata Vieira, and Rafael H. Bordini Predicting Plan Failure by Monitoring Action Sequences and Duration
ADCAIJ: Advances in Distributed Computing and Articial Intelligence Journal Regular Issue, Vol. 6 N. 4 (2017), 55-69 eISSN: 2255-2863 - http://adcaij.usal.es Ediciones Universidad de Salamanca - CC BY NC DC 
Panisson, A. R., Freitas, A., Schmidt, D., Hilgert, L., Meneguzzi, F., Vieira, R., and Bordini, R. H., 2015. Arguing About Task Reallocation Using Ontological Information in Multi-Agent Systems. In 12th International Workshop on Argumentation in Multiagent Systems.

Pearl, J., 1988. Probabilistic Reasoning in Intelligent Systems: Networks of Plausible Inference. Morgan Kaufmann.

Pereira, R. F., Oren, N., and Meneguzzi, F., 2017. Landmark-Based Heuristics for Goal Recognition. In Proceedings of the 32nd AAAI Conference on Artificial Intelligence.

Pynadath, D. V. and Wellman, M. P., 1995. Accounting for Context in Plan Recognition, with Application to Traffic Monitoring. In Proceedings of the Eleventh Conference on Uncertainty in Artificial Intelligence, UAI'95, pages 472-481. Morgan Kaufmann Publishers Inc., San Francisco, CA, USA. ISBN 1-55860-385-9.

Ramírez, M. and Geffner, H., 2009. Plan Recognition as Planning. In Proc. IJCAI, pages 1778-1783.

Rich, C. and Sidner, C. L., 1997. COLLAGEN: when agents collaborate with people. In Proceedings of the first international conference on Autonomous agents, AGENTS '97, pages 284-291. ACM, New York, NY, USA. ISBN 0-89791-877-0. doi:10.1145/267658.267730.

Sukthankar, G., Goldman, R. P., Geib, C., Pynadath, D. V., and Bui, H. H., editors, 2014. Plan, Activity, and Intent Recognition: Theory and Practice. Elsevier. ISBN 978-0123985323.

Sukthankar, G. and Sycara, K., 2011. Activity Recognition for Dynamic Multi-Agent Teams. ACM TIST, 3(1):18. Ullmer, B. and Ishii, H., 2000. Emerging Frameworks for Tangible User Interfaces. IBM Syst. J., 39(3-4): 915-931. ISSN 0018-8670. doi:10.1147/sj.393.0915.

Waern, A., 1996. Recognising Human Plans: Issues for Plan Recognition in Human-Computer Interaction. $\mathrm{Ph} . \mathrm{D}$. thesis, The Royal Institute of Technology and Stockholm University.

Giovani P. Farias, Ramon Fraga Pereira, Lucas W. Hilgert, Felipe Meneguzzi, Renata Vieira, and Rafael H. Bordini Predicting Plan Failure by Monitoring Action Sequences and Duration
ADCAIJ: Advances in Distributed Computing and Articial Intelligence Journal Regular Issue, Vol. 6 N. 4 (2017), 55-69 elSSN: 2255-2863 - http://adcaij.usal.es Ediciones Universidad de Salamanca - CC BY NC DC 
